\title{
Low-Complex Reliable Communications between Wireless Network-Nodes
}

\author{
Alois M.J. Goiser \\ Institute of Telecommunications \\ University of Technology Vienna, Austria \\ Email: alois.goiser@tuwien.ac.at
}

\begin{abstract}
We present a low-complex blind interference reduction scheme embedded in the receiver to enhance correlative data detection. The key element is a statistically controlled adaptive nonlinearity prior to correlation. This add-on feature guaranties reliable communication between network-nodes in any type of noise and/or interference. Additionally the concept provides accurate position measurements to make services flexible and intelligent.
\end{abstract}

Keywords-Capture-Effect, Interference Combating, Nonlinear Signal Processing, Continuous-Wave Interference, Non-Gaussian Noise, Wireless Application, Robust Communication, Reliable Communication, Spread-Spectrum.

\section{INTRODUCTION}

$\mathbf{W}$ $\mathrm{E}$ assume that in future industrial networks the communication between nodes will be wireless. The advantage, beside others, of wireless technology is its flexibility to create and change existing services. The disadvantage of wireless is its air-interface, which is subject to any electromagnetic interference. Especially in industrial applications it is desirable to use robust schemes to establish reliable links [1]. To achieve this goal for digital communications, we suffer from the so called capture-effect, which is specific for low-complex digital philosophies and degrades the performance significantly. We present an adaptive nonlinearity that is controlled by magnitude and level-crossing statistics to enable it to cope with the capture-effect. The presented add-on interference reduction scheme exploits only statistical parameters derived from the received signal and makes no assumptions about the channel state nor it makes any attempt to analyze it. So it is a blind scheme. The advantages of blind-schemes are: (1) No channel estimates are necessary, avoiding estimation-errors, avoiding adaptation problems in high dynamic interference environments. (2) Needs no return channel to the transmitting node. The consequence of the blindness-feature is, that it is not allowed to make any assumption about the interference environment. We test the investigated scheme with interfering waveforms ranging from broadband, modeled as AWGN (Gaussian noise), to narrowband, modeled as continuous-wave (CW) interference (non-Gaussian noise). The only assumption that is made about the interference is that the mechanism is additive on the magnitude scale and permanently present.

In the following, we will show that the proposed scheme is always better than a conventional one-bit quantized digital correlation detector. We assume this structure as the compet- assumption). We refer to it as $H L$-detector. The investigated scheme is a baseband blind adaptive interference reduction (BAIR) scheme and we refer to it as BAIR-detector.

The motivation for this paper and the positioning in the literature is treated in section VI.

\section{CAPTURE EFFECT}

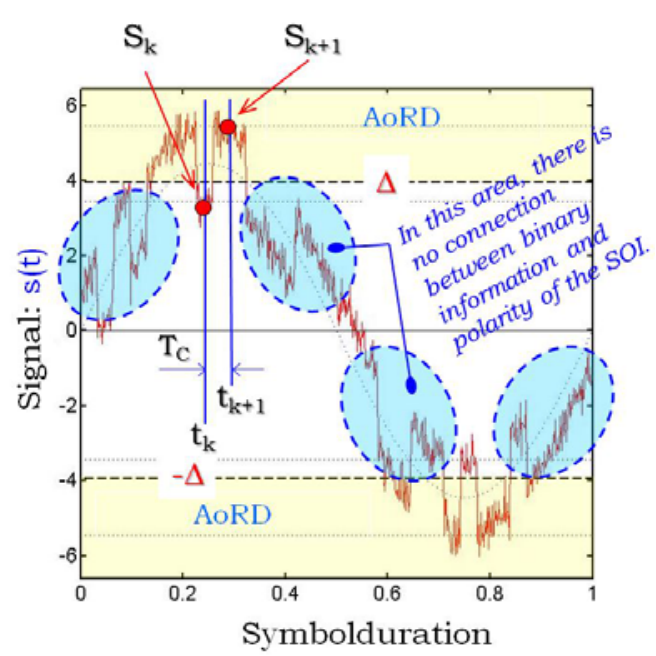

Fig. 1. Received signal at baseband with dominating CW-interference. SOI ... PN-sequence, SONI .... AWGN plus CW. AoRD ... Area of Reliable Decisions, $S_{k} \ldots$ sample at time instant $t_{k}, \pm \Delta \ldots$ symmetric magnitude threshold-pair

The nature of the capture-effect is that a weak desired signal (SOI ... signal of interest) is shift in magnitude by a strong undesired signal (SONI ... signal of no interest) to render the polarity-information in the SOI. This situation is sketched in Fig.1. It can easily verified that the HL-detector suffers from the capture-effect, because the chip-decision threshold is the zero-level. Both samples in Fig.1 lead to a positive chipdecision, but the sample $S_{k}$ corresponds to a negative chip. As will be pointed out in the next sections the investigated BAIRdetector compares the signal samples with the threshold $\Delta$ and makes both decisions to correct chip-decisions.

\section{InVESTIGATED S YSTEM LAYOUT}

The investigations are done at the physical layer with baseband-signals and focus on the interference reduction capability and their performance description, so the usual error control coding for reliable communication is not included. 


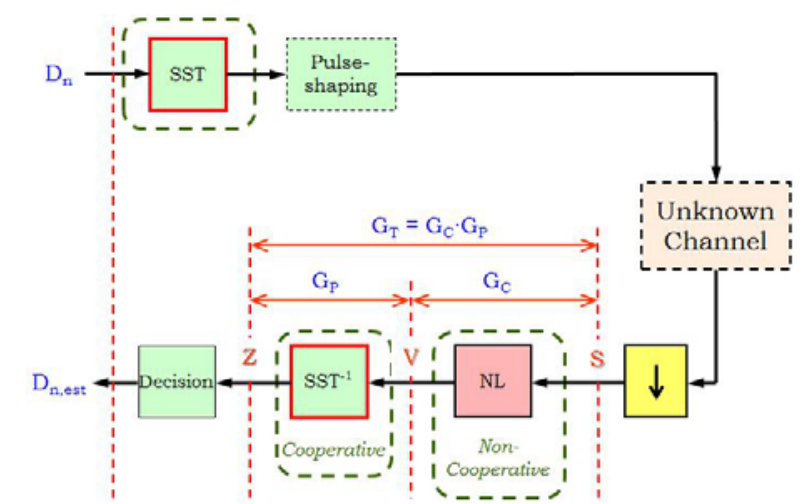

Fig. 2. Generic structure of an efficient communication complex with integrated interference reduction. SST ... spread-spectrum technology, NL ... nonlinearity, $G_{p} \ldots$ processing gain, $G_{c} \ldots$ conversion gain, $G_{T} \ldots$ total gain, $S, V, U \ldots$ random variables, $Z \ldots$ decision variable.

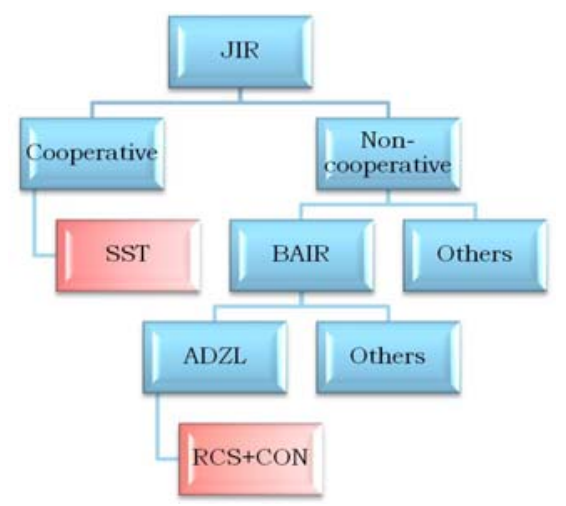

Fig. 3. Overwiew of Joint Interference Reduction (JIR). SST ... spreadspectrum technology, BAIR ... blind adaptive interference reduction, ADZL ... adaptive dead-zone limiter, RCS ... reliable chip-selector, CON ... threshold control.

Implementing interference reduction schemes in conventional communication systems are investigated by many authors, see section VI.

We propose a generic structure to combine cooperative and non-cooperative interference reduction in one communication complex as sketched in Fig.2. The combination between cooperative and non-cooperative interference reduction schemes offer the possibility to maximize physical layer performance. We refer to this combination of interference reduction schemes as Joint Interference Reduction (JIR). An overview is presented in Fig.3. The investigated system is embedded in the generic structure and sketched in Fig.4: The cooperative part corresponds to the spread-spectrum modulation and the BAIRconcept corresponds to the non-cooperative part. The BAIRconcept is realized with the Adaptive Dead-Zone Limiter (ADZL).

The BAIR-concept affects the signaling of the communication complex. If we have no network, only a single transmitter/receiver-pair (single link assumption) is active and operate in an exclusive frequency range and is faced with uncorrelated broadband and narrowband interference. For that assumption we need only one PN-signature for signaling with phase-reversal-keying modulation. In a network with many nodes, each node uses a distinct orthogonal signature. Due to the fact that not all samples of one symbol are included

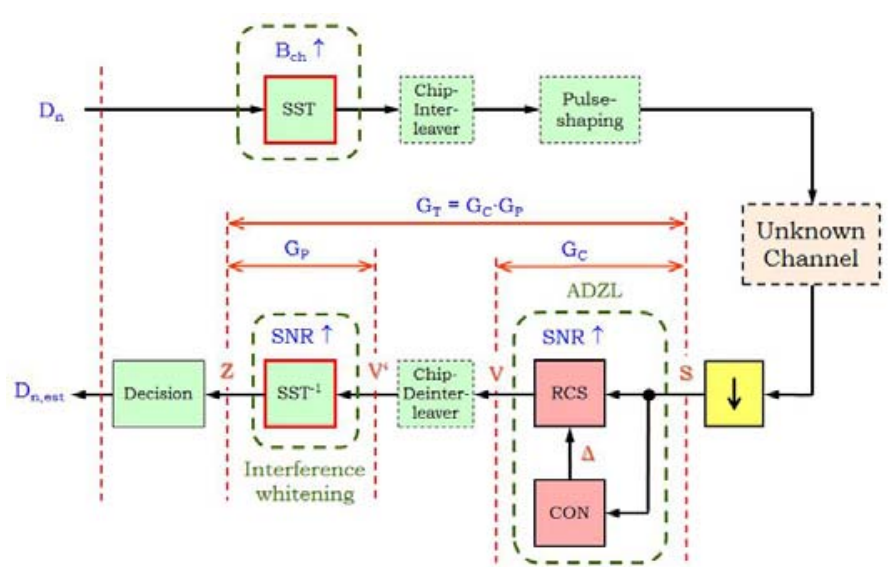

Fig. 4. Investigated communication complex - BAIR-detector. SST ... spreadspectrum technology, RCS ... reliable chip selector, CON ... threshold control module, ADZL ... Adaptive Dead Zone Limiter, $B_{c h} \ldots$ channel bandwidth.

in the decision process (presented in the next section) we expect a slight degradation due to the leakage in orthogonality between the active waveforms. To reduce the degradation we introduce a chip-interleaver after spread-spectrum modulation. The reverse operation at the receiver is placed between the ADZL-module and the correlator. The interleaver randomizes the erased samples to statistically independent events.

\section{JOINT INTERFERENCE REDUCTION}

As mentioned previously we refer to joint interference reduction when cooperative interference reduction and noncooperative interference reduction work together to maximize physical layer performance. In our transceiver concept in Fig.4 we implement the cooperative part in spread-spectrum technology, more specific as direct-sequence signal and the noncooperative part as ADZL. We gain from both concepts the advantages. From the spread-spectrum technology we exploit the whitening property with the processing gain $G_{p}$. From the ADZL we exploit the capability to overcome the captureeffect and achieve the conversion gain $G_{c}$. Both gains together achieve the total gain $G_{T}$ in (1). The total gain enhance the SOI to SONI power-ratio from the received signals $\left(\mathrm{SINR}_{s}\right)$. The symbol-error-probability (SEP) is given in (2).

$$
\begin{gathered}
G_{T}=G_{p} \cdot G_{c} \\
\mathrm{SEP}=\frac{1}{2} \cdot \operatorname{erfc}\left(\sqrt{\frac{G_{c} G_{p}}{2} \cdot \mathrm{SINR}_{s}}\right)
\end{gathered}
$$

\section{A. Spread-Spectrum Technology}

The nature of the interference reduction capability offered from the spread-spectrum technology is based on the whitening property. The whitening procedure is definitely a cooperative concept, because the transmitter designs the channel signal in such a way that the receiver has the possibility to reduce the harm of the interfering waveforms. It is also a blind philosophy, because no attempt is made to analyze the interference situation. The pseudo-random (PN) nature of the direct-sequence signal is responsible for the whitening effect [2]. The performance measure is the processing gain (3) and 
directly related to the number of chips $(L)$ available in the direct-sequence signal. The mechanism is that the SONI is chopped by the chips into small time segments (chip-duration) with pseudo-random polarities. The signal is known to the receiver and all the polarity-changes from the SOI are reversed while at the same time the polarity changes are introduced in the SONI making the SONI, regardless of their original nature, noise-like. The noise-like signal is averaged out by the subsequent integration operation.

$$
G_{p}=L \quad \text {.. Processing Gain }
$$

Including the spread-spectrum concept into the interference reduction task offers an additional property to make nodes more intelligent. Due to the quasi white nature of the SOI the autocorrelation function is impulse like. This property offers accurate measurements for position location systems or services.

\section{B. Blind Adaptive Interference Reduction}

The first step in the detection process in any digital communication receiver is to sample the received waveform. In our concept the SOI is a direct-sequence signal and the minimum sampling rate is the chip-rate. To cope with non-Gaussian noise we split the symbol decision into L equal sub-decisions (chip-decisions). So a wrong sub-decision counts only for one wrong sub-decision out of many sub-decisions. That limits the interference power.

In our concept we go one step further. We derive a measure to decide if a sub-decision is more reliable than other subdecisions. The device for this task is referred to as reliable chip selector (RCS). So the RCS decides if a sub-decision is feed forward to the final symbol decision or erased. The mapping from a received signal sample to a chip-decision is sketched in Fig.5. A dead-zone limiter has the appropriate characteristic to realize the mapping and is sketched in Fig.6.

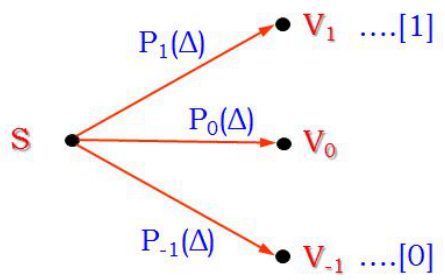

Fig. 5. Mapping of the RCS: Received signal sample maps to chip-decision.

The information content of the polarity of the SOI must be retrieved from the received signal. That is done by erasing the samples in the unreliable area (mainly occupied by the SONI, area of unreliable decisions ... AoUD) to emphasize the samples from the reliable area (mainly occupied by the SOI, area of reliable decisions ... AoRD). The most important task is to distinguish both regions (reliable/unreliable) accurately. To achieve that we exploit results from the level-crossingtheory. We show that the level-crossing theory offers the desired information to separate the regions reliably. This is based on the assumption that the hot magnitude area is the area where level-crossings occur frequently. That means that the magnitude of the peak of the level-crossings dictates the separation threshold.

The adjustment of the threshold $\Delta$ in the ADZL to its optimum location is done in the CON-module, sketched in Fig.4. A detailed description of the optimization process for threshold adjustment is given in [3].

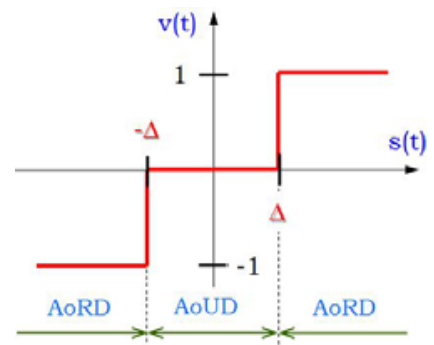

Fig. 6. Adaptive dead-zone limiter. AoRD ... Areo of reliable decisions, AoUD ... Areo of unreliable decisions.

$G_{c}=\frac{\operatorname{SINR}_{v}}{\operatorname{SINR}_{s}} \quad$.... Conversion Gain

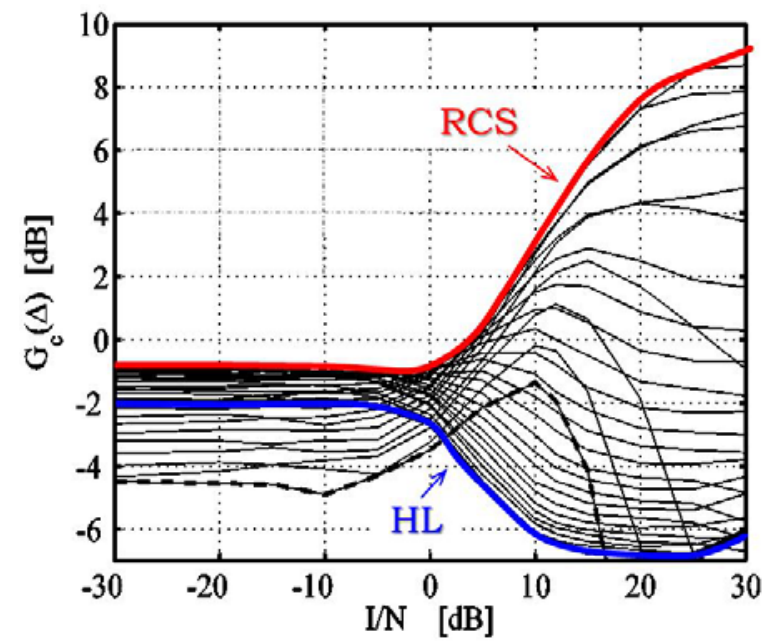

Fig. 7. Comparison of the conversion-gains for RCS and HL.

The performance of the RCS is quantified with the conversion-gain. The conversion gain is defined as the ratio of the power-ratios between SOI and SONI at the output respective to the input (4). The conversion-gain is sketched in Fig.7 and compared with the counterpart the HL-device. The mean conversion-gain for one sample, sampled at the chip-rate, is drawn against the power-ratio of the ratio of the SONIs (I ... power of the $\mathrm{CW}, \mathrm{N} \ldots$ power of the AWGN), while the power-ratio of the SOI respective the SONI is kept fixed at $-15 \mathrm{~dB}$ (poor signal condition). A detailed evaluation of the conversion-gain can be found in [4]. The significant improvement for dominating $\mathrm{CW}$-interference is remarkable. In roughly pure Gaussian noise we note an advantage in power-gain of about $1.5 \mathrm{~dB}$ (left edge in Fig.7) and in nonGaussian noise ( $\mathrm{CW}$-interference) we achieve a power-gain of about $15 \mathrm{~dB}$ (right edge in Fig.7). It is also notable that the competing HL-detector is completely captured by the nonGaussian noise and unable to handle it, resulting in a drop of power-gain of about $7 \mathrm{~dB}$. Also in dominating Gaussian noise 
the HL-detector experience a $2 \mathrm{~dB}$ gap in conversion-gain. The advantage in conversion-gain of the BAIR-dectector compared to the HL-detector ranges from $1.5 \mathrm{~dB}$ to $22 \mathrm{~dB}$.

\section{PERFORMANCE}

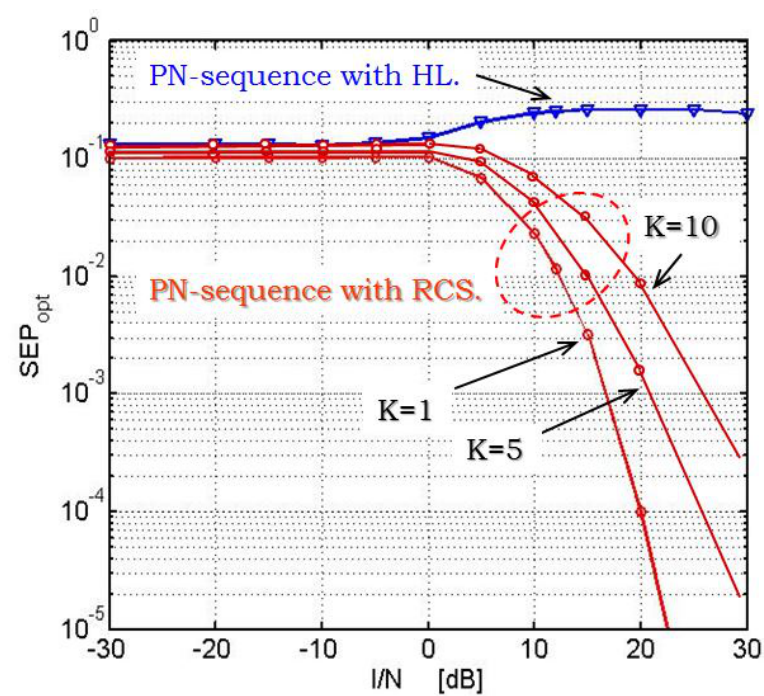

Fig. 8. Performance comparison: $\mathrm{PN}$-signature with HL-detection $(\mathrm{K}=1)$ and $\mathrm{PN}$-signature with RCS assisted detection for K active links.

The performance of the investigated communication complex is presented with the conversion-gain of the RCS defined in (4) and sketched in Fig.7 and the symbol-error-probability, sketched in Fig.8 for the same signal conditions as used in the calculation for the conversion-gain. The processing-gain of the direct-sequence spread-spectrum system is $15 \mathrm{~dB}$. The power ratio of the SOI to the SONI is $-15 \mathrm{~dB}$ and fixed in the calculation. The power of the SONI consists of the power of the WGN (white Gaussian noise) combined with the power of the CW-interference (non-Gaussian nature). The power ration of $I / N$ indicates if Gaussian or non-Gaussian interference dominates. The field of the conversion-gain curves in Fig.7 are sketched from dominating WGN (left side) to dominating CWinterference (right side) with field-parameter $\Delta$ (the threshold of the ADZL shown in Fig.6). So for $\Delta=0$ we see the conversion-gain of the HL-detector. For the HL-device the conversion-gain ranges from about $-2 \mathrm{~dB}(\mathrm{WGN})$ to about $-7 \mathrm{~dB}(\mathrm{CW})$ which is in agreement with the theory [4]. The RCS-device adjust the threshold $\Delta$ for varying interference compositions always to its optimum location. The optimum threshold-location is defined as the location for which the maximum of the conversion gain is achieved and represented in Fig.7 with the RCS-curve. Details of the threshold-control can be found in [3].

The symbol-error-probability in Fig.8 is sketched for the RCS-detector indicating that the threshold of the ADZL is always adjusted to its optimum location (RCS-curve in Fig.7). The HL-detector is completely captured by the strong $\mathrm{CW}$-interference and useless. The improvement in symbolerror-probability for the RCS-detector, originated from the conversion-gain for dominating $\mathrm{CW}$-interference, is obvious. Details about the performance evaluation for a single link $(\mathrm{K}=1)$ structure can be found in [4].
For multi-node communications at the same location, each transceiver-pair has its unique signature, drawn from an orthogonal PN-signal set. The degradation due to the collisions of $\mathrm{K}$ active sequences is presented in Fig.8. The results are derived from simulations under the assumption that all active links are on the same power-level. To maintain quasiorthogonality between the SOIs, which is essential for multiuser communications, we introduce chip-interleaving. That guarantee that no long runs of erased-chip decisions occur.

\section{Vi. Contribution to Literature}

The brief review is done with respect to interference reduction in general and level crossing statistics specifically. Unfortunately a bulk of literature is available in the military community and not available. A lot of papers on interference reduction are written by Larry Milstein. The topic of many of his papers are related to adaptive filtering of a narrowband interfering signal from a broadband user signal. This situation occur in a direct-sequence spread-spectrum communication link. He exploits the different nature of the wanted and unwanted signal. He wrote an excellent tutorial paper [57] on this topic in 1988. An other tutorial paper [45] was written by Laster and Reed covering interference mitigation techniques for wireless applications. A survey of robust signal processing was written by Kassam and Poor [41]. The basic properties of the spread-spectrum technology are pointed out in [59], [49], [8]. The robust nature of the spread-spectrum technology was investigated in [28], [29]. Narrowband interference reduction concepts using adaptive notch-filters were investigated among others from Milstein, Doherty, Green, Reed, Davis, Krieger, Haimovich, Vadhari [13], [34], [47], [48], [35], [57], [73], [65], [23], [36], [18], [19], [17], [31]. This type of interference reduction is able to remove the power of the unwanted signal close to 100 percent and leave the wanted signal roughly undistorted. The algorithms are usually complex and sometimes suffer from stability problems. Especially when the dynamic of the estimated signal is large. The method of transform domain processing is used from Davidovici and others [16], [22], [66], [64]. This method is straight forward but usually complex especially if the narrowband interference changes its frequency and magnitude rapidly. The paper from Krinsky focuses on burst type error patterns [44].

Robust signal processing topics are dominated by publications from Kassam, Poor and others [39], [40], [76]. The algorithms derived in these papers are sometimes complicated and need a lot of computation power. The focus is mainly on amplitude statistics. The coding approach is based on bandwidth expansion related to the redundancy included in the information stream. A book written by Gallager serves as excellent reference [20].

The multiple access scheme using the spread-spectrum technology is the code-division multiple access [72]. This is not a collision avoidance system and therefore interference reduction leads directly to a better overall performance. This type of interference is definitely non-Gaussian in its origin. Many papers and books are devoted to this problem without additional interference reduction processing. But it was recognized very early that interference reduction can improve the 
performance significantly [58], [43], [60], [75], [74]. From this early results the multiuser detection concept evolve. The scientists that proposed multiuser detection are Verdu, Poor, Lupas and many other papers are devoted to this topic [46], [71]. The most efficient use of multiuser detection is through jointly optimal decoding, which is prohibitively complex for many users. Suboptimum solutions were investigated to reduce the complexity but suffer from performance degradation.

A fewer number of communication scientists investigate memoryless nonlinearities to reduce the power of the unwanted signal. Nonlinearities in communication engineering usually degrade the performance [63], [30] and are not used to enhance the signal-to-noise ratio. This unwanted nonlinear effects result from irregular operating conditions, like overload and saturation effects. Pioneering communication engineers in the 1950's have recognized that certain nonlinearities can achieve an advantage and gain in signal-to-noise ratio. In general, this type of interference reduction technique also corrupts the wanted signal. But the ratio of reduction in power is in favor to the wanted signal. Usually this methods are fast and independent of the dynamic of the unwanted signal. The first application was the so called bandpass-limiter investigated by Davenport, Cahn and Blachman [15], [14], [38], [9], [10], [11], [50], [12], [37] and dates back to 1961. The bandpasslimiter was used for bandpass signals and is located in the input section of the receiver.

The exploitation of nonlinear interference reduction processing in addition to the robust nature of the spread-spectrum technology was investigated in [24], [25], [26], [27], [28], [29], [1]. The adaptation of the characteristic of an analog memoryless nonlinearity to the behavior of the unwanted signal, for non spread-spectrum systems, was investigated by Higbie in [32]. His approach works best for slowly changing interference. Amoroso and other authors have exclusively used magnitude statistics to adapt discontinuous nonlinearities [5], [6], [7]. That concept suffer from a static error if the composition of the interference changes.

The inclusion of the information of the level-crossings to the information content of the magnitude statistics bridge the gap to optimum and fast adaptation using simple and low complex memoryless nonlinearities prior to spread-spectrum detection [3], [4]. That previous analysis are done for the single-link case. In this paper we include the multi-link case and the chipinterleaving which is essential for multi-link communication to maintain quasi-orthogonality between the signatures during the detection process.

A very important topic for all non-blind interference reduction schemes is to know the behavior of the interference. Usually the interference is manifested in the detection process as a signal. Therefore the study of the behavior of the interference is a study of the unwanted signal and takes place in the signal domain. It is in nature a random signal and information from such a signal can only be derived by statistical means. The focus was exclusively on magnitude statistics, because the detector processes magnitudes to make the decision. To derive the statistical behavior we have to study the interference source or interfering sources if there are more sources contributing to the interference. This leads us to characterize the interference and classify it. David Middleton gave an interview with Vincent Poor [55]. In this discussion he pointed out that communication scientists have to focus more on the physics of interference to derive models for the interfering process that fit to real applications. He wrote many key papers on the characterization and classification of interference [51], [52], [53]. In a very remarkable paper David Middleton [54] wrote: Thus, in signal processing overall, as well as in model-building we have evolved from the Gaussian noise world of the 1950's to the general non-Gaussian noise environment of the 1980's, where Gaussian noise is now recognized as an important but special case of real-world interference. He resumes further that we need adaptivity.

Investigations for impulsive interference are mainly done by David Middleton and Spaulding [68], [69], [70], [56]. This type of interference is non-Gaussian and nonlinearities are included to limit the influence of the interference impulses.

The first attention on level-crossing theory was payed by S.O.Rice in his pioneering paper [61] about a mathematical description of random noise. His intention was to model the random noise as accurate as possible. He calculated the level-crossing rate and the time between crossings. The levelcrossings are suitable to detect frequency modulated signals. The main application of level-crossing statistics today is to compute the mean duration of a fade in a mobile environment [33]. There is no publication available on level-crossings for interference reduction and/or using the level-crossings for nonlinear detection. The most rigorous investigations on level-crossings are done in the book from Kedem [42]. But applications are mainly devoted to frequency estimation.

Also recognized by S.O. Rice was that complexity is also an real engineering interest [62]. He presented the first curves of error probability versus delay and complexity. Slepian and Wyner mentioned that in their honorable paper [67].

\section{CONCLUSION}

In this paper we have investigated a robust and reliable communication scheme with the following advantages: It is from low complexity, that results in low power consumption and low implementation costs. The control loop for the threshold adjustment is fast converging and stable. The BAIR-module works in line (information flow), so no feedback is required and it is always in a secure state. It outperforms its counterpart the hard-limiter detector in any type of interference. In Gaussian noise we achieve about $1.5 \mathrm{~dB}$ power-gain and in non-Gaussian noise we achieve power-gains up to $15 \mathrm{~dB}$. An add on is the accurate position measurement for intelligent position based services.

\section{REFERENCES}

[1] A. Goiser, S. Khattab, "Robust and Reliable Communication Meets Future Mobile Communication Demands", Proceedings ICAS2010, 2010.

[2] R.L. Pickholtz, D.L. Schilling, L.B. Milstein, "Theory of SpreadSpectrum Communications - A Tutorial", IEEE Transactions on Communications, COM-30, Nr.5, 855-884, 1982, "Revisions", IEEE Transactions on Communications, COM-32, Nr.2, 1984.

[3] A. Goiser, S. Khattab, G. Fassl, U. Schmid, "A New Robust Interference Reduction Scheme for Low Complexity Direct-Sequence SpreadSpectrum Receivers: Optimization”, Proceedings NexComm2010, 2010. 
[4] A. Goiser, S. Khattab, G. Fassl, U. Schmid, "A New Robust Interference Reduction Scheme for Low Complexity Direct-Sequence SpreadSpectrum Receivers: Performance", Proceedings NexComm2010, 2010.

[5] F.Amoroso, J.Bricker, "Increasing the Up-Link CW Interference Immunity of Non-Coherent Direct Sequence Pseudonoise (DSPN) Reception with On-Board Processing.", International Journal of Satellite Communications, Vol.11, 107-118, 1993.

[6] F.Amoroso, "Adaptive A/D converter to suppress co-channel constant envelope interference in a mobile digital link.", Telecommunication Systems, Vol.2, 109-119, (1993).

[7] F. Amoroso, R. Monzingo, "Adaptive A/D Converter for Improved DS/SS Jam Resistance", Transactions on Aerospace and Electronic Systems, Vol.34, Nr.3, 810-816, 1998.

[8] P.W. Baier, "Spread-Spectrum-Technik und CDMA", telecom praxis, 5/95, 9-14, 1995.

[9] N.M. Blachman, "Band-Pass Nonlinearities", IEEE Transactions on Information Theory, IT-10, Nr.2, 162-164, 1964

[10] Nelson M. Blachman, "Detectors, Bandpass Nonlinearities, and Their Optimization: Inversion of the Chebyshev Transform", IEEE Transactions on Information Theory, IT-17, Nr.4, 398-404, 1971.

[11] N.M. Blachman, "Noise and its Effect on Communication", KriegerPublishing, Malabar, 1982.

[12] N.M. Blachman, "Optimum Memoryless Bandpass Nonlinearities", Proceedings IEEE-Milcom'93, 263-267, 1993.

[13] M.J. Bouvier Jr., "The Rejection of Large CW Interferers in SpreadSpectrum Systems", IEEE-Transactions on Communication, COM-26, Nr.2, 254-256, 1978.

[14] C.R. Cahn, "A Note on Signal-to-Noise Ratio in Band-Pass Limiters", IEEE Transactions on Information Theory, IT-7, Nr.1, 39-43, 1961

[15] W. Davenport Jr., "Signal-to-Noise Ratios in Band-Pass-Limiters", Journal of Applied Physics, Vol.24, Nr.6, 720-727, 1953.

[16] S. Davidovici, E.G. Kanterakis, "Narrow-Band Interference Rejection Using Real-Time Fourier Transforms", IEEE Transactions on Communications, COM-37, Nr.7, 713-722, 1989.

[17] M.E. Davis, L.B. Milstein, "Anti-jamming properties of a DS-CDMA equalization filter", Proceedings IEEE-Milcom'93, 1008-1012, 1993.

[18] J.F. Doherty, "A constrained LMS algorithm for interference rejection" , Proceedings IEEE-Milcom'92, 696-700, 1992.

[19] J.F. Doherty, H. Stark, "Direct-Sequence Spread-Spectrum Narrowband Interference Rejection Using Property Restoration", IEEE Transactions on Communications, COM-44, Nr.9, 1197-1204, 1996.

[20] R. Gallager, "Inforamtion Theory and Reliable Communication", Wiley \& Sons, ISBN 978-0471290483, 1968.

[21] W.A. Gardner, "Cyclostationarity in Communications and Signal Processing", IEEE Press, ISBN 0-7803-1023-3, 1994.

[22] J. Gevargiz, P. Das, L.B. Milstein, "Adaptive Narrow-band Interference Rejection in a DS Spread-Spectrum Intercept Receiver Using Transform Domain Signal Processing Techniques", IEEE Transactions on Communications, COM-37, Nr.12, 1359-1366, 1989.

[23] C.D. Green, J.H. Reed, N.M. Yuen, T.C. Hsia, "An optimal receiver using a time-dependent adaptive filter", Proceedings IEEE-Milcom'89, 1989.

[24] A. Goiser, "Enhancing the Signal-to-Interference Ratio in Digital DirectSequence Spread-Spectrum Receivers", International Journal of Wireless Information Networks, Plenum-Press, August, 173-186, 1997.

[25] A. Goiser, "Threshold Synchronization of the Adaptive Interference Reduction Scheme in Low Complexity Digital Direct-Sequence SpreadSpectrum Receivers", Proceedings IEEE-Milcom'97, 74-78, 1997.

[26] A. Goiser, "A Heuristic Approach to Higher Order Statistical Models for a Combined Process of a Direct-Sequence Signal in Additive White Gaussian Noise and Continuous Wave Interference after an Adaptive 2-bit ADC Nonlinearity", Proceedings IEEE-Milcom'97, 580-588, 1997.

[27] A. Goiser, "A Fast Multi User Detector for DS/CDMA-Networks using Partial Sequence Estimation", Proceedings IEEE-Milcom'99, 34.7, 1999.

[28] A. Goiser, "About the Robust Nature of Spread-Spectrum Systems", Proceedings IEEE-ISSTA'02, 465-470, 2002.

[29] A. Goiser, "Exploiting the Robust Nature of Spread-Spectrum Technology for Future Wireless Applications", e \& i Elektrotechnik und Informationstechnik, 23-27, 2004.

[30] A.H. Haddad, "Nonlinear Systems: Processing of Random Signals Classical Analysis", ISBN: 0-470-33848-2, Halsted Press, 1975.

[31] A. Heimovich, A. Vadhri, "Rejection of narrow-band interferences in PN spread-spectrum systems using an eigenvalue analysis approach", Proceedings IEEE-Milcom'94, 1007-1011, 1994.

[32] J.H. Higbie, "Adaptive Nonlinear Suppression of Interference", Proceedings IEEE-Milcom'88, 23.3.1-23.3.9., 1988.
[33] J. Ilow, P. Venkatasubramanian, "Applications of Level Crossing Theory to Clipping Noise Characterization in Filtered OFDM Signals.", (2007).

[34] R.A. Ilits, L.B. Milstein, "Performance Analysis of Narrow-Band Interference Rejection Techniques in DS Spread-Spectrum Systems", IEEE Transactions on Communications, COM-32, Nr.11, 1169-1177, 1984.

[35] R.A. Ilits, L.B. Milstein, "An Approximate Statistical Analysis of the Widrow LMS Algorithm with Application to Narrow-Band Interference Rejection", IEEE Transactions on Communications, COM-33, Nr.2, 121130, 1985.

[36] R.A. Ilits, "A GLRT-Based Spread-Spectrum Receiver for Joint Channel Estimation and Interference Suppression", IEEE Transactions on Communications, COM-37, Nr.3, 277-288, 1989.

[37] P. Jain, N. Blachman, P. Chapell, "Interference Suppression by Biased Nonlinearities", IEEE Transactions on Information Theory, IT-41, Nr.2, 496-507, 1995 .

[38] J.J. Jones, "Hard-Limiting of Two Signals in Random Noise", IEEE Transactions on Information Theory, IT-9, Nr.1, 34-42, 1963.

[39] S.A. Kassam, "Optimum Quantization for Signal Detection", IEEE Transactions on Communications, COM-25, Nr.5, 479-484, 1977.

[40] S.A. Kassam, T.L. Lim, "Coefficient and Data Quantization in Matched Filters for Detection", IEEE Transactions on Communications, COM-26, Nr.1, 124-131, 1978

[41] S.A. Kassam, H.V. Poor, "Robust Techniques for Signal Processing: A Survey.", IEEE Proceedings, Vol.73, Nr.3, 433-481, 1985.

[42] B. Kedem, "Time Series Analysis by Higher Order Crossings", IEEE Press, ISBN: 0-87942-299-8, 1994.

[43] R. Kohno, H. Imai, M. Hatori, S. Pasupathy, "An Adaptive Canceller of Cochannel Interference for Spread-Spectrum Multiple-Access Communication Networks in a Power Line", IEEE Journal on Selected Areas in Communications, SAC-8, Nr.5, 691-699, 1990.

[44] D. Krinsky, A. Haddad, C .Lee, "An Adaptive Direct-Sequence SpreadSpectrum Receiver for Burst Type Interference", IEEE Selected Areas in Communications, SAC-13, Nr.1, 59-70, 1995

[45] J.D. Laster, J.H. Reed, "Interference Rejection in Digital Wireless Communications", IEEE Signal Processing Magazine, 37-62, 1997.

[46] R. Lupas, S. Verdu, "Linear Multiuser Detectors for Synchronous CodeDivision Multiple-Access Channels", IEEE Transactions on Information Theory, IT-35, Nr.1, 123-136, 1989.

[47] E. Masry, "Closed-Form Analytical Results for the Rejection of NarrowBand Interference in PN Spread-Spectrum Systems - Part I: Linear Prediction Filters", IEEE-Transactions on Communication, COM-32, Nr.8, 888-896, 1984.

[48] E. Masry, "Closed-Form Analytical Results for the Rejection of NarrowBand Interference in PN Spread-Spectrum Systems - Part II: Linear Interpolation Filters", IEEE-Transactions on Communication, COM-33, Nr.1, 10-19, 1985.

[49] J.L. Massey, "Information Theory Aspects of Spread-Spectrum Communications", IEEE Proceedings of the Third International Symposium on Spread-Spectrum Techniques \& Applications, 16-21, 1994.

[50] Bruce F. McGuffin, "The Effect of Bandpass Limiting on A Signal in Wideband Noise", Proceedings IEEE-Milcom'92, 206-210, 1992.

[51] D. Middleton, "Statistical-Physical Models of Man-Made and Natural Radio Noise", OT-Report 76-86, 1999.

[52] D. Middleton, "Statistical-Physical Models of Electromagnetic Interference", IEEE Transactions on Electromagnetic Compatability, EMC-19, 106-127, 1977.

[53] D. Middleton, "Canonical and Quasi-Canonical Probability Models of Class A Interference", IEEE Transactions on Electromagnetic Compatability, EMC-25, 76-106, 1983.

[54] D. Middleton, "S.O. Rice and the Theory of Random Noise: Some Personal Recollections", IEEE Transactions on Information Theory, IT34, Nr.6, 1367-1373, 1988

[55] D. Middleton, "A Conversation with David Middleton.", Vincent Poor. IEEE Information Theory - News Letter, 1995.

[56] D. Middleton, "Non-Gaussian Noise Models in Signal Processing for Telecommunications: New Methods and Results for Class A and Class B Noise Models", IEEE Transactions on Information Theory, IT-45, Nr.4, $1129-1149,1999$

[57] L.B. Milstein, "Interference Rejection rejection Techniques in SpreadSpectrum Communications.", IEEE Proceedings, Vol.76, No.6, 1988.

[58] L.B. Milstein, J. Wang, "Interference Suppression for CDMA Overlays of Narrowband Waveforms", IEEE Proceedings of the Third International Symposium on Spread-Spectrum Techniques \& Applications, 61-68, 1994.

[59] R.L. Pickholtz, D.L. Schilling, L.B. Milstein, "Theory of SpreadSpectrum Communications - A Tutorial.”, IEEE Transactions on Commu- 
nications, COM-30, Nr.5, 855-884, 1982, "Revisions", IEEE Transactions on Communications, COM-32, Nr.2, 1984.

[60] W. Pietsch, A. Goiser, "A New Receiver Operating in Multiple Access Interference Related to the Near Far Problem", Proceedings IEEEMilcom'90, 47.3.1.-47.3.4., 1990.

[61] S. O. Rice, "Mathematical analysis of random noise", Bell Syst. Tech. J., vol. 23, pp. 282-332, 1944; Vol.24, pp. 46-156, 1945.

[62] S. O. Rice, "Communication in the presence of noise - Probability of error for two encoding schemes.", Bell Syst. Tech. J., Vol. 29, pp. 60-93, 1950.

[63] G.H. Robertson, "Performance Degradation by Postdetector Nonlinearities", Bell System Technical Journal, 407-414, 1968.

[64] S.D. Sandberg, "Adapted Demodulation for Spread-Spectrum Receivers which Employ Transform-Domain Interference Excision", IEEE Transactions on Communications, Vol.43, Nr.9, 2502-2510, 1995.

[65] G.J. Saulnier, P. Das, L.B. Milstein, "Suppression of Narrow-Band Interference in a PN Spread-Spectrum Receiver Using a CTD-Based Adaptive Filter", IEEE Transactions on Communications, COM-32, Nr.11, 12271232, 1984

[66] G.J. Saulnier, "Suppression of Narrowband Jammers in a SpreadSpectrum Receiver Using Transform-Domain Adaptive Filtering", IEEE Selected Areas in Communication, Vol.10, Nr.4, 742-749, 1992.

[67] D. Slepian, A.D. Wyner, "S.O. Rice's Contributions to Shannon Theory", IEEE Transactions on Information Theory, IT-34, Nr.6, 1367-1373, 1988.

[68] A.D. Spaulding, D. Middleton, "Optimum Reception in an Impulsive Interference Environment-Part I: Coherent Detection", IEEE Transactions on Communications, COM-25, Nr.9, 910-923, 1977.

[69] A.D. Spaulding, D. Middleton, "Optimum Reception in an Impulsive Interference Environment-Part II: Noncoherent Reception", IEEE Transactions on Communications, COM-25, Nr.9, 924-934, 1977.

[70] A.D. Spaulding, "Locally Optimum and Suboptimum Detector Performance in a Non-Gaussian Interference Environment", IEEE Transactions on Communications, COM-33, Nr.6, 1985.

[71] S. Verdu, "Multiuser Detection", Cambridge University Press, ISBN 0521-59373-5, 1998

[72] A. Viterbi, "CDMA-Principles of Spread-Spectrum Communication", Adison-Wesley Wireless Communications Series, 1995.

[73] Y.C. Wang, L.B. Milstein, "Rejection of Multiple Narrow-Band Interference in Both BPSK and QPSK DS Spread-Spectrum Systems", IEEE Transactions on Communications, COM-36, Nr.2, 195-204, 1988.

[74] J. Wang, L.B. Milstein, "Adaptive LMS Filters for Cellular CDMA Overlay Situations", IEEE Journal on Selected Areas in Communications, SAC-14, Nr.8, 1548-1559, 1996.

[75] P. Wei, R. Zeidler, W. Ku, "Adaptive Interference Suppression for CDMA Overlay Systems", IEEE Selected Areas in Communications, SAC-12, Nr.9, 1510-1523, 1994.

[76] W.R. Wu, F.F. Yu, "New Nonlinear Algorithms for Estsimating and Suppressing Narrowband Interference in DS Spread-Spectrum Systems", IEEE Transactions on Communications, COM-44, Nr.4, 508-515, 1996.

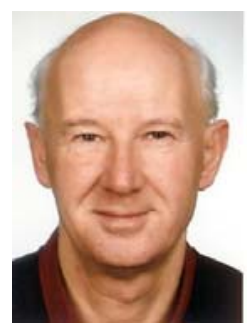

Alois M.J. Goiser Currently he holds the position of an associated professor at the University of Technology Vienna at the Institute of Telecommunications. He has published about forty papers in the field of interference reduction and spread-spectrum technology. His primary research interest deals with robust and reliable communications. Specifically he is interested in nonlinear receiver design, interference reduction, spread-spectrum communication, wireless communication and research for future communication systems. He has published one book about spread-spectrum communications and three contributions to books about wireless communications. 